\title{
Laboratory Testing of the Innovative Low-Cost Mewar Angithi Insert for Improving Energy Efficiency of Cooking Tasks on Three-Stone Fires in Critical Contexts
}

\author{
Jacopo Barbieri $^{1, *(\infty}$, Fabio Parigi ${ }^{2}$, Fabio Riva ${ }^{1}\left(\mathbb{D}\right.$ and Emanuela Colombo ${ }^{1}$ \\ 1 Department of Energy, Politecnico di Milano, via Lambruschini 4/a, 20156 Milano, Italy; \\ fabio.riva@polimi.it (F.R.); emanuela.colombo@polimi.it (E.C.) \\ 2 Sustainable Grill, Via Palach 10, 20090 Segrate, Italy; info@sustainablegrill.com \\ * Correspondence: jacopo.barbieri@polimi.it
}

Received: 24 October 2018; Accepted: 7 December 2018; Published: 11 December 2018

check for updates

\begin{abstract}
Currently, about 2.7 billion people across the world still lack access to clean cooking means. Humanitarian emergencies and post-emergencies are among the most critical situations: the utilization of traditional devices such as three-stone fires have a huge negative impact not only on food security but also on the socio-economic status of people, their health and the surrounding environment. Advanced Cooking Stoves may constitute better systems compared to actual ones, however, financial, logistic and time constraints have strongly limited the interventions in critical contexts until now. The innovative, low-cost Mewar Angithi insert for improving energy efficiency of three-stone fires may play a role in the transition to better cooking systems in such contexts. In this paper, we rely on the Water Boiling Test 4.2.3 to assess the performances of the Mewar Angithi insert respect to a traditional three-stone fire and we analyse the results through a robust statistical procedure. The potentiality and suitability of this novel solution is discussed for its use in critical contexts.
\end{abstract}

Keywords: Improved Cooking Stove; Mewar Angithi; humanitarian settings; Water Boiling Test

\section{Introduction}

\subsection{Access to Energy in Critical and Humanitarian Settings}

Nowadays, about 2.7 billion people still have no access to clean cooking, with almost 30\% and $65 \%$ of whom living in sub-Saharan Africa and developing Asia, respectively. Among them, more than 2.3 billion people still rely on traditional solid biomass (e.g., fuelwood, agricultural waste, animal dung), while the others mostly on kerosene and coal [1]. In such countries, cooking indoors with a traditional open fire (also called three-stone fire, TSF) is among the causes respiratory illness (e.g., respiratory infections, chronic obstructive pulmonary disease, lung cancer, cardiovascular disease and eye irritation [2]), which contributes to the premature death of more than 2.5 million people per year. The problem mostly affects women and children [3]. In addition, illness due to respiratory infections has been identified as one of the most common cause of absenteeism from school in some countries in sub-Saharan Africa [4]. From an environmental point of view, the utilization of traditional biomass is a recognized contributor to deforestation and land degradation. Even if agriculture and timber industry are known as the major drivers of large scale deforestation, firewood collection and charcoal making for domestic uses can have significant impacts on local ecosystems, especially in densely populated areas [5-7]. Biomass burning in traditional cookstoves has also been found to be 
responsible for about $20 \%$ of global black carbon emissions, which are an important contributor to global warming [8].

The situation of people living in humanitarian settings, including refugees and Internally Displaced People (IPDs), is particularly problematic. More than 65.6 million people worldwide have been forced to leave their homes by wars, conflicts and natural disasters by the end of 2016 [9]. Proper access to sustainable energy resources and adequate cooking systems is one of the major challenges displaced people are facing daily. Despite the evidence that more than $80 \%$ of displaced people have access to traditional biomass only and $90 \%$ do not have access to electricity [10], the problem of energy in humanitarian contexts is usually underestimated by the humanitarian sector [11], due to the fact that other priorities are considered, including the provision of food and medical treatments.

Displaced people can have a tremendous impact on the surrounding ecological system, affecting the future lives of both refugees and local people and creating conflicts between hosted and hosting communities. For example, in north-western Tanzania, the influx of half million displaced people caused the depletion of trees for $5 \mathrm{~km}$ radius from the camps in just 6 months and the deforested area reached $10 \mathrm{~km}$ radius in 1 year [12]. The amount of firewood globally consumed by displaced people in refugee camps, leads to the loss of 64,000 acres of forest per year and to an estimated release of 13 million tons of $\mathrm{CO}_{2}$ into the atmosphere [7] (around $1 \%$ of the total $\mathrm{CO}_{2}$ emission from fuel combustion of the African continent). The utilization of traditional fuels also causes the emission of enormous quantities of pollutants at the local level, which is considered a contributor to the premature death for some 20,000 displaced people each year [13]. Usually women and young girls are in charge of the gathering of firewood, spending many hours daily. They are obligated to walk further as the trees diminish, exposing them to safety risks $[3,7,14]$. Refugee camps are running out of charcoal and firewood and the lack of fuel is affecting food security. For example, in Kounoungou and Mille camps in Chad, $35 \%$ of refugees had to skip meals and $28 \%$ ate food undercooked due to fuel scarcity. Also, selling as much as $25 \%$ of food rations for purchasing cooking fuel is common in Nakivale refugee camp in Uganda [13]. Similar figures have been depicted also in North Darfur, where $80 \%$ of refugees were forced to sell part of the food received from international organizations in order to purchase firewood and cook the remaining rations [7]. In this framework, the Inter-Agency Standing Committee indicates three complementary actions to overcome energy poverty among displaced people [15]: (i) decrease the fuel needed to prepare a typical meal; (ii) promote sustainable biomass collection; and (iii) provide alternative solutions for both fuel and cooking tools. Improved Cooking Stoves (ICSs) are among the most promoted solutions in the short- and mid-term to contribute to points (i) and (iii): several designs of ICSs were deployed in the last decades taking into account different socio-cultural and economic aspects. Despite local circumstances, the main challenges that aid organizations are facing to deploy and maintain ICSs programs over time are: (i) the scarcity and durability of financial resources and the expensive price of commercial ICSs; (ii) the fact that refugees are often willing to sell not only food ratios but also ICSs that have been donated (a quick cash back option to purchase fuel or other higher priority goods, such as mobile phones or lighting devices $[16,17])$; and (iii) the fact that ICSs that require a strong modification of traditional cooking practices are often characterized by low levels of acceptance $[18,19]$.

For these reasons, simpler and low-cost cooking solutions may represent a viable option to reach a larger number of displaced people more effectively, especially during the emergency phase of the humanitarian response. Therefore, this work provides a first evaluation of the performances of an innovative simple insert, which may improve the performances of traditional cooking systems in critical contexts. In fact, the characteristics of such insert, as described in the next paragraph, positively address the main challenges previously described.

\subsection{The Mewar Angithi Insert: Concept and Characteristics}

The Mewar Angithi (MA) insert was firstly designed by a team of experts in Rajasthan, India, in late 2014, with the aim of developing a low-cost device to be inserted in traditional three-stone 
fires. Subsequently, the developers introduced the MA insert also in Ghana and Kenya [20,21], finding a reduction of the amount of firewood needed to accomplish daily food preparation. Therefore, the team of developers created the Sustainable Grill (http:/ / sustainablegrill.com/) association for the improvement and dissemination of the technology in critical contexts of the world.

The MA insert is a simple metal grill, costing around $\$ 1$, that is easy to be inserted in traditional cooking systems, such as TSFs or mud stoves (Figures 1 and 2), improving thermal efficiency and lowering emissions.

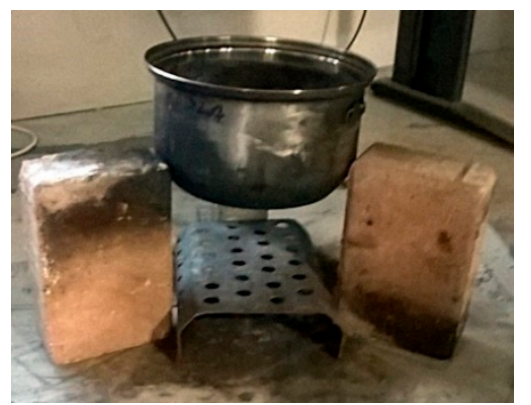

Figure 1. The Mewar Angithi (MA) inserted in a three-stone fire.

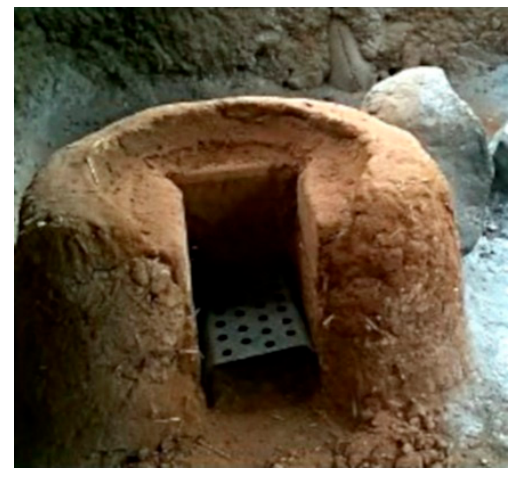

Figure 2. The MA inserted in a horseshoe mud stove.

In a traditional cooking system, firewood is usually placed directly on the ground, which increases the heat losses through conductive transfer to the ground, restricts the airflow through the fuel and interferes with the proper mixing of fuel and combustive agent. These sources of losses decrease the thermal efficiency. Therefore, the cooking activity requires a huge quantity of fuel. Similarly, the inefficient combustion increases the quantity of PM and CO emissions released in the air [22,23]. The insertion of the MA insert in a TSF or mud stove allows to lift the firewood off the ground and to ensure a constant air supply to the bottom. In particular, the MA insert overcomes the well-known problem of traditional cooking stoves whereby thermal efficiency decreases gradually during the cooking session due to the lack of oxygenation caused by suffocation of the fire by accumulated ash and embers produced during combustion of the fuel.

Despite the simple shape and the high temperature reached by the insert during the combustion process, the insert does not show particular criticalities regarding its durability, with very limited degradation of the material. However, further experimental campaigns are planned by the developers for testing this particular aspect.

A particular strength of the MA insert, is the fact that its utilization only slightly modifies the traditional cooking system and cooking process, which increases the probability of its successful implementation, by limiting as much as possible its impact on local social and cultural environment $[20,21]$. Furthermore, the MA insert can be easily locally manufactured by using common tools for working metal and providing basic trainings to local artisans (Figure 3). 

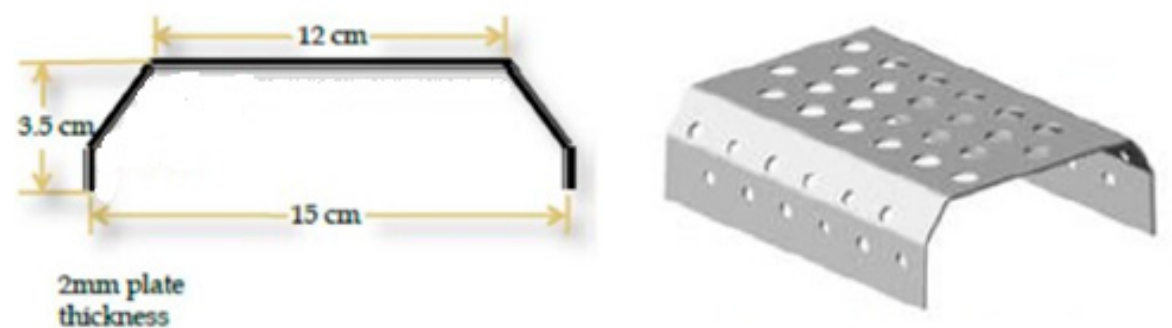

Figure 3. Technical drawings of the MA insert.

The aim of this study is to perform a quantitative evaluation of the performances of the MA insert, through laboratory testing following the Water Boiling Test, which has been extensively used in similar studies, including, for example, [22,24-26]. Despite the recent findings showing that real field performances may differ substantially from lab results [27], we decided to adopt this protocol to carry out a first step evaluation, with the idea of performing further tests in the field in case of positive results from the lab (it is worth to note that at the time of the experimental campaign, the new ISO 19867-1:2018-Clean cookstoves and clean cooking solutions-Harmonized laboratory test protocols -Part 1: Standard test sequence for emissions and performance, safety and durability was not still established). It is worth noting that some preliminary tests were carried out on the insert directly in the field $[20,21]$, however without following a formalized protocol with standardized indicators and a rigorous statistical analysis of the results. Based on that, this study stands on the request of the developers to carry out an experimental campaign in a controlled environment in order to obtain results comparable with other similar studies.

We performed the evaluation by comparing the results obtained from a traditional TSF versus a TSF coupled with the MA insert, operated under the same controlled conditions in laboratory. This choice allows drawing significant conclusions on the performances of the TSF with the MA insert compared to the TSF alone, even if the performances detected in the lab, in absolute terms, may differ from those in the field.

\section{Materials and Methods}

\subsection{Materials Testing Protocol}

The Water Boiling Test (WBT) protocol version 4.2.3 [28] was used to evaluate the performances of the MA insert in this study. The WBT 4.2.3 consists in a sequence of three phases (Figure 4): (i) Cold Start High Power: fresh water at ambient temperature contained in a pot, at ambient temperature, is brought to the boiling point on the stove to be tested using pre-weighted fuel. Standard amount of water to be boiled is 2.5 or 5 litres, depending on the pot and stove size. (ii) Hot Start High Power: while the stove is still hot, the pot is replaced with a new one at ambient temperature containing fresh water, at ambient temperature, that is brought to the boiling point. (iii) Simmering: the hot water from the previous phase is kept at a temperature between the boiling point and the boiling point minus six Celsius degrees $\left(100_{-6}^{0}{ }^{\circ} \mathrm{C}\right)$ for $45 \mathrm{~min}$.

We selected the indicators used in the study from among the ones officially recognised by IWA, in order to make the selection consistent with the ISO/IWA 11:2012 guidelines [29]. The chosen indicators are: (i) High Power Thermal Efficiency (\%); (ii) Low Power Specific Consumption Rate (MJ/min/L); (iii) High Power, Low Power and Total Fuel consumption (g); (iv) High Power PM $\left(\mathrm{mg} / \mathrm{MJ}_{\mathrm{d}}\right)$ and Low Power PM $(\mathrm{mg} / \mathrm{min} / \mathrm{L})$; $(\mathrm{v})$ High Power CO $\left(\mathrm{g} / \mathrm{MJ} \mathrm{d}_{\mathrm{d}}\right.$ ) and Low Power $\mathrm{CO}(\mathrm{g} / \mathrm{min} / \mathrm{L})$. 


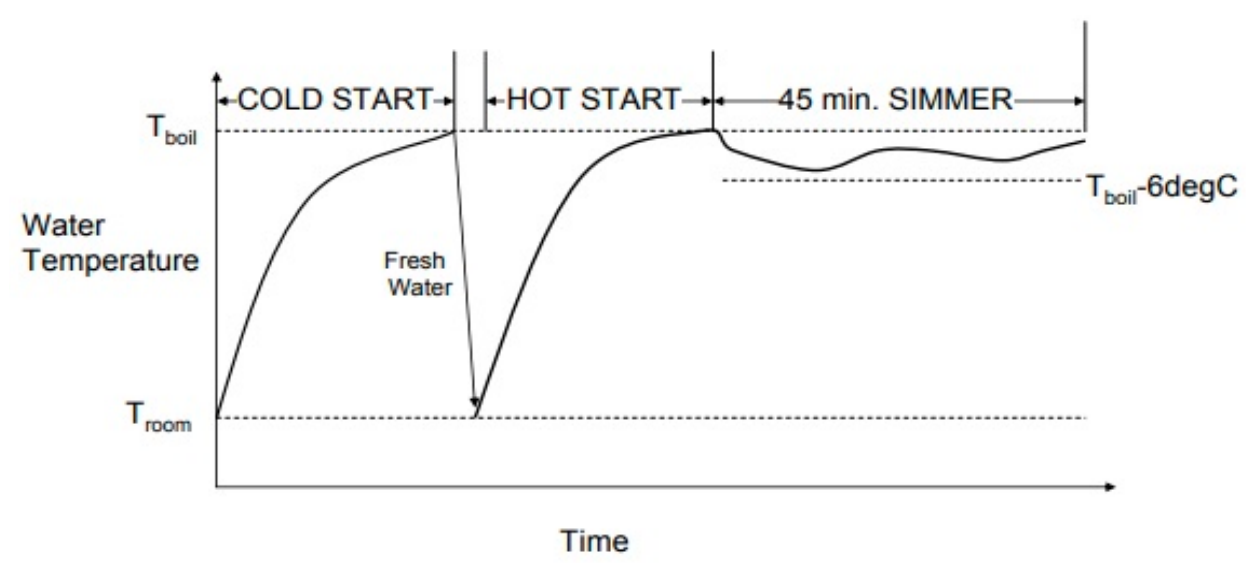

Figure 4. Graphical representation of the Water Boiling Test (WBT) phases (Source: [28]).

It is worth noting that High Power indicators are calculated as the average measurements obtained during the phase (i) and (ii) of the test (cold start and hot start), while Low Power indicators are obtained from the phase (iii) (simmering).

\subsection{Laboratory Equipment}

Data for evaluating the performances of the MA insert compared to TSF have been acquired using the Portable Emission Monitoring System (PEMS) by Aprovecho ${ }^{\circledR}$ (Cottage Grove, OR, USA). Table 1 reports the characteristics of the main sensors included in the PEMS (PEMS Technical Specifications Sheet 8.23 .17 by Aprovecho Research Center.):

Table 1. Portable Emission Monitoring System (PEMS) sensors.

\begin{tabular}{lll}
\hline \multicolumn{1}{c}{ CO } & \multicolumn{1}{c}{$\mathbf{C O}_{2}$} & \multicolumn{1}{c}{ PM } \\
\hline Type: Electrochemical cell & Type: Non-Dispersive Infrared & \\
Range: $0-5000 \mathrm{ppm}$ & Range: 0-10,000 ppm & Type: Red laser scattering photometer \\
Accuracy: $25 \mathrm{ppm}$ & Accuracy: $75 \mathrm{ppm}$ or $10 \%$ & Range: 0-60,000 ug $/ \mathrm{m}^{3}$ \\
Resolution: $1 \mathrm{ppm}$ & (whichever is greater) & Resolution: $25 \mathrm{ug} / \mathrm{m}^{3}$ \\
Response time: $\mathrm{T} 90<25 \mathrm{~s}$ & Resolution: $2 \mathrm{ppm}$ & Response time: $1 \mathrm{~s}$ \\
& Response time: T90 < $2 \mathrm{~min}$ & \\
\hline
\end{tabular}

In addition, the following measurement tools have been used:

- Electronic weight scale, resolution $1 \mathrm{~g}$ up to $5 \mathrm{~kg}, 2 \mathrm{~g}$ from 5 to $10 \mathrm{~kg}$;

- Wood moisture meter, electric resistance type, sensitivity $1 \%$ up to $30 \%$ of moisture content, $2 \%$ between $30 \%$ and $60 \%, 4 \%$ from $60 \%$ up to $90 \%$;

- Air moisture (humidity) meter, resolution $0.1 \%$, accuracy $\pm 3 \%(30-99 \% \mathrm{RH}), \pm 5 \%(10-30 \% \mathrm{RH})$;

- $\quad$ Thermocouple type K.

\subsection{Statistical Analysis}

We carried out a robust statistical analysis of data following the procedure described in Lombardi et al. [30,31].

We gave particular attention to the minimum number of replicates of the WBT necessary to obtain reliable results. Considering that three replicates are not sufficient [32,33], we adopted the incremental convergence criterion as defined in Reference [30], considering the idea that the minimum number of tests is achieved only when the addition of a new set of data from a further replicate does not significantly affect the standard deviation of the whole set of indicators. In practical terms:

$$
\left(\sigma_{n}-\sigma_{n-1}\right)-\left(\sigma_{n-1}-\sigma_{n-2}\right) \leq k^{*}
$$


where $\sigma$ is the standard deviation of the dataset for each indicator obtained in the $n$-th replicate of the experiment and $k^{*}$ a selected threshold (10\% in this study).

\section{Results}

The results of the experimental campaign are presented in Table 2. The incremental convergence criterion previously described was applied to set the minimum number of replicates. For two indicators, convergence was reached only at the 10-th replicate. We therefore performed 10 replicates of the WBT for both the devices, although for some indicators less replicates would have been enough to fulfil the criterion (1).

Table 2. Results of laboratory testing for each selected indicator.

\begin{tabular}{cccc}
\hline Indicator & TSF & MA Insert & $\begin{array}{c}\text { Percentage Difference } \\
\text { (MA Insert vs TSF) (\%) }\end{array}$ \\
\hline Number of test replicates (-) & 10 & 10 & \\
High Power Thermal Efficiency (-) & $0.0951(0.0173)$ & $0.1274(0.0194)$ & $34.07^{* * *(31.79)}$ \\
Low Power Specific Consumption Rate (MJ/min/L) & $0.1413(0.0311)$ & $0.1199(0.0173)$ & $-15.14^{* *}(22.32)$ \\
High Power Fuel Consumption (g) & $1101.2680(268.0091)$ & $791.5806(143.5347)$ & $-28.12^{* * *}(21.81)$ \\
Low Power Fuel Consumption (g) & $639.2175(128.7607)$ & $507.0024(83.1822)$ & $-20.68^{* * *}(20.61)$ \\
Total Fuel Consumption (g) & $1740.4860(354.8518)$ & $1298.5830(191.8221)$ & $-25.39^{* * *}(18.78)$ \\
High Power PM (mg/MJd) & $178.8463(76.1455)$ & $112.8398(43.3735)$ & $-36.911^{* *}(36.19)$ \\
Low Power PM (mg/min/L) & $3.8971(1.3743)$ & $2.0293(1.2452)^{+}$ & $-47.93^{* * *}(36.85)^{++}$ \\
High Power CO (g/MJd) & $10.7817(4.6559)$ & $9.7703(2.7739)$ & $-9.38(46.83)$ \\
Low Power CO (g/min/l) & $0.2473(0.0714)$ & $0.2045(0.0453)^{+}$ & $-17.32(30.09)^{++}$ \\
\hline
\end{tabular}

Standard Deviation in brackets. ${ }^{* *}$ and ${ }^{* * *}$ indicate significance at the $95 \%$ and $99 \%$ level, respectively. ${ }^{+}$Normality hypothesis rejected for this indicator (Shapiro-Wilk's normality test). ${ }^{++}$Significance level based on Wilcoxon rank-sum test.

It is worth noting that the Shapiro-Wilk's test was run on the dataset to check the normality hypothesis. Normality hypothesis was confirmed in all cases, except for the case of Low Power CO and Low Power PM for the MA insert. Therefore, non-parametrical test of significance (Wilcoxon rank-sum) was applied for these indicators and the conservative approach of Chebyshev's inequality was applied to evaluate confidence intervals in place of $\mathrm{t}$-student distribution.

We compared the results obtained for the TSF and the TSF equipped with the MA insert by calculating the relative difference among the two cooking systems.

The first two selected indicators (Thermal Efficiency and Specific Consumption Rate) provide information about energy performances of the compared systems. According to the WBT protocol, we evaluate the Thermal Efficiency during the High Power (HP) phase, while the Specific Consumption Rate during the Low Power (LP) Phase. The utilization of the MA insert increments the efficiency of about 3 percentage points in absolute terms. In relative terms, this means that HP Thermal Efficiency is increased by $34.1 \%$ on average $(p<0.01)$, with a shift from $9.51 \%(\mathrm{SD}=1.73, \mathrm{Ue}=1.00)$ for the case of TSF to $12.74 \%$ ( $\mathrm{SD}=1.94, \mathrm{Ue}=1.13$ ) when TSF is coupled to the MA insert (in this study we select Confidence Interval (CI) equal to $90 \%$ unless otherwise specified. Ue is calculated according to the standard definition, as explained in [31]).

As expected, the Specific Consumption Rate follows an opposite trend: during the LP phase, the indicator is equal to $0.14 \mathrm{MJ} / \mathrm{min} / \mathrm{L}(\mathrm{SD}=0.03$, $\mathrm{Ue}=0.02$ ) for the case of TSF, while $0.12 \mathrm{MJ} / \mathrm{min} / \mathrm{L}$ $(\mathrm{SD}=0.02, \mathrm{Ue}=0.01)$ when TSF is coupled to the MA insert, which corresponds to a relative decrease of $15.1 \%$ on average $(p<0.04)$.

Total Fuel Consumption is reduced by $25.39 \%$ (i.e., about $440 \mathrm{~g}$ in absolute terms, $p<0.01$ ) on average when the MA insert is used $(1740.49 \mathrm{~g}, \mathrm{SD}=354.85$, $\mathrm{Ue}=205.70 ; 1298.58 \mathrm{~g}$, $\mathrm{SD}=191.82$, $\mathrm{Ue}=111.20$ respectively for the case of TSF and TSF with MA insert). In fact, the reduction is more substantial during the HP phase, while the improvement is less accentuated during the LP phase.

The different savings attributable to HP and LP phase do not surprise if we think about the different operations performed in the two phases. During the LP phase, in fact, the tester is required to keep the water temperature as constant as possible. In order to do so the fire is accurately controlled 
and the power of the flame is lowered with respect to the HP phase, whose goal is to increase the temperature as much as possible.

Looking at the emissions, PM emission rates are reduced in both HP and LP phases (the reduction is significant at $C I=99 \%$ based on Wilcoxon rank-sum test $(p=0.003)$ ), respectively by $36.91 \%$ and $47.93 \%$ on average ( $p<0.02$ and $p<0.01$, respectively). It is worth noting that the two indicators have different measurement units. In absolute terms, PM during the HP phase is equal to $178.85 \mathrm{mg} / \mathrm{MJd}$ $(\mathrm{SD}=76.15, \mathrm{Ue}=44.14)$ for the case of TSF alone and to $112.48 \mathrm{mg} / \mathrm{MJd}(\mathrm{SD}=43.37, \mathrm{Ue}=25.14)$ for the case of TSF with MA insert. During the LP phase, instead, the values are respectively equal to $3.90 \mathrm{mg} / \mathrm{min} / \mathrm{L}(\mathrm{SD}=1.37, \mathrm{Ue}=0.80)$ and $2.03 \mathrm{mg} / \mathrm{min} / \mathrm{L}(\mathrm{SD}=1.25, \mathrm{Ue}=3.94$; Low Power PM data are not normally distributed. For this reason, we calculated the expanded uncertainty Ue using the Chebyshev's inequality and $C I=90 \%)$. It is worth noting that for the case of the MA the Ue is overextended as an effect of the utilization of Chebyshev's inequality.

As regards $\mathrm{CO}$ emissions, a reduction of emission rates for the case of TSF with MA insert compared to the case of TSF alone is not proven by the statistical analysis of the results. In other words, based on the experimental campaign here presented, the two systems present the same rates of CO emissions.

\section{Discussion and Conclusions}

In this study we present the results of a laboratory campaign to assess the performances of the Mewar Angithi (MA) insert, a simple low-cost metal grid that can be inserted in traditional cooking stoves such as three-stone fires and mud stoves to improve their performances.

The statistical analysis, based on the experimental results of the Water Boiling Test 4.2.3, shows that the MA insert brings significant improvements in terms of high-power thermal efficiency $(+34 \%)$, PM emissions ( $-37 \%$ during High Power phase and $-48 \%$ during Low Power phase) and total fuel consumption $(-25 \%)$, while no significant improvement is found as regards CO emissions.

It is worth underlying that this study only represents a first step of evaluation, while an experimental campaign in the field may constitute a second one. In fact, even if the Water Boiling Test is still the most widely adopted test for the evaluation of Improve Cooking Stoves performances, recent studies have shown that real performances in the field can substantially differ from lab results [27]. In this perspective, on the one hand some promising preliminary results from the utilization of the MA insert in Kenya, seem to be in line with our findings, with improvements of thermal efficiency in the range $25-40 \%$ when the MA insert is coupled to three-stone fires and horseshoe mud stove [20]. On the other hand, our findings differ from those from another preliminary assessment of the MA insert carried out in India [21], suggesting that it is necessary to further assess the performances of the insert in different field conditions.

In any case, it is clear that a traditional stove coupled with the MA insert still remains in the category of basic cooking technologies, with lower performances compared to most improved and modern cooking devices (e.g., gasifiers, insulated stoves, LPG and electric cookers) and high levels of pollutant emissions. For this reason, the MA insert should be considered as a temporary solution to reduce the negative impacts associated to the utilization of traditional cooking systems. On the other hand, the very low cost (around $\$ 1$ and ease of local manufacturing of the MA insert, makes it a ready-to-use option in contexts where other solutions would take longer to be implemented, or would not be feasible due to financial constraints. In particular, the MA insert may be successfully introduced in emergency or post-emergency settings, such as refugee camps and informal settlements, where the supply of fuels other than firewood is not feasible on the short-term and limited resources represent a bottleneck for the provision of more sophisticated devices. For example, looking at the case of Goudoubo refugee camp in Burkina Faso, hosting more than 10,000 people, and of Kakuma I refugee camp in Kenya, hosting roughly 14,000 people, it is estimated that more than $18 \mathrm{~kg} /$ day of firewood is consumed by each family cooking with TSF based on the data reported by the Moving Energy Initiative [16,34]. On average, this leads to a consumption of about $600 \mathrm{ton} / \mathrm{month}$ and $650 \mathrm{ton} / \mathrm{month}$, 
respectively. If the families in the camps were provided with the MA insert, in between 40-265 tons and 43-287 tons of firewood respectively would be saved each month, with a consequent potential reduction of the overall families' income expenditure.

Author Contributions: Conceptualization, J.B. and F.P.; Formal analysis, J.B.; Investigation, J.B. and F.R.; Methodology, J.B. and F.R.; Supervision, E.C.; Writing—original draft, J.B.; Writing—review \& editing, J.B., F.P., F.R. and E.C.

Funding: This research received no external funding.

Acknowledgments: The authors gratefully acknowledge the support of Giada Meroni and Marco Cerri in performing the experimental campaign.

Conflicts of Interest: The authors declare no conflict of interest.

\section{References}

1. IEA. World Energy Outlook 2018; IEA: Paris, France, 2018.

2. IEA. Energy Access Outlook 2017: From Poverty to Prosperity; IEA: Paris, France, 2017; Volume 94.

3. Putti, V.R.; Tsan, M.; Mehta, S.; Kammila, S. The State of the Global Clean and Improved Cooking Sector; World Bank: Washington, DC, USA, 2015.

4. Sovacool, B.K. The political economy of energy poverty: A review of key challenges. Energy Sustain. Dev. 2012, 16, 272-282. [CrossRef]

5. Arnold, J.E.M.; Köhlin, G.; Persson, R. Woodfuels, livelihoods, and policy interventions: Changing perspectives. World Dev. 2006, 34, 596-611. [CrossRef]

6. Mandelli, S.; Barbieri, J.; Mattarolo, L.; Colombo, E. Sustainable energy in Africa: A comprehensive data and policies review. Renew. Sustain. Energy Rev. 2014, 37, 656-686. [CrossRef]

7. Barbieri, J.; Riva, F.; Colombo, E. Cooking in refugee camps and informal settlements: A review of available technologies and impacts on the socio-economic and environmental perspective. Sustain. Energy Technol. Assess. 2017, 22, 194-207. [CrossRef]

8. Ramanathan, V.; Carmichael, G. Global and regional climate changes due to black carbon. Nat. Geosci. 2008, 1, 221-227. [CrossRef]

9. UNHCR. Global Trends_Forced Displacement in 2015; UNHCR: Geneva, Switzerland, 2016.

10. Moving Energy Initiative. Energy Consumption of Refugees and Displaced People Database; Moving Energy Initiative: London, UK, 2018.

11. Burrows, G.; Ballard-Tremeer, G.; Garside, B.; Jones, D.; Rai, K.; Severi, L. Energy in Emergency Settings; Boiling Point: Chislehurst, UK, 2016.

12. Shepherd, G. The Impact of Refugees on the Environment and Appropriate Response; Humanitarian Practice Network: London, UK, 1995.

13. Lahn, G.; Grafham, O. Heat, Light and Power for Refugees. Saving Lives, Reducing Costs; Chatham House: London, UK, 2015.

14. Köhlin, G.; Sills, E.O.; Pattanayak, S.K.; Wilfong, C. Energy, Gender and Development: What Are the Linkages? Where Is the Evidence? World Bank: Washington, DC, USA, 2011.

15. IASC. Guidelines for Integrating Gender-Based Violence Interventions in Humanitarian Action; IASC: Geneva, Switzerland, 2015.

16. Corbyn, D.; Vianello, M. Prices, Products and Priorities Meeting Refugees' Energy Needs; Chatham House: London, UK, 2018.

17. Bellanca, R. Sustainable Energy Provision among Displaced Populations: Policy and Practice; Chatham House: London, UK, 2014.

18. Confino, J.; Paddison, L. Cookstove designs are failing the poorest communities. The Guardian, 7 February 2014.

19. Lehne, J.; Blyth, W.; Lahn, G.; Bazilian, M.; Grafham, O. Energy services for refugees and displaced people. Energy Strateg. Rev. 2016, 13-14, 134-146. [CrossRef]

20. Parigi, F.; Del Viscio, M.; Amicabile, S.; Testi, M.; Rao, S.; Udaykumar, H.S. High efficient Mewar Angithi stove testing in rural Kenya. In Proceedings of the 2016 7th International Renewable Energy Congress (IREC), Hammamet, Tunisia, 22-24 March 2016; pp. 1-6. 
21. Udaykumar, H.S.; Kindig, A.; Rao, S.; Del Viscio, M.; Kukillaya, V.; Panwar, N.L.; Sharma, D. How a simple inexpensive device makes a three-stone hearth as efficient as an improved cook-stove. Solut. J. 2015, 6, 53-60.

22. Jetter, J.J.; Kariher, P. Solid-fuel household cook stoves: Characterization of performance and emissions. Biomass Bioenergy 2009, 33, 294-305. [CrossRef]

23. Shen, G.; Gaddam, C.K.; Ebersviller, S.M.; Vander Wal, R.L.; Williams, C.; Faircloth, J.W.; Jetter, J.J.; Hays, M.D. A Laboratory Comparison of Emission Factors, Number Size Distributions, and Morphology of Ultrafine Particles from 11 Different Household Cookstove-Fuel Systems. Environ. Sci. Technol. 2017, 51, 6522-6532. [CrossRef] [PubMed]

24. Ojo, K.D.; Soneja, S.I.; Scrafford, C.G.; Khatry, S.K.; LeClerq, S.C.; Checkley, W.; Katz, J.; Breysse, P.N.; Tielsch, J.M. Indoor particulate matter concentration, water boiling time, and fuel use of selected alternative cookstoves in a home-like setting in rural Nepal. Int. J. Environ. Res. Public Health 2015, 12, 7558-7581. [CrossRef] [PubMed]

25. Obeng, G.Y.; Mensah, E.; Ashiagbor, G.; Boahen, O.; Sweeney, D.J. Watching the smoke rise up: Thermal efficiency, pollutant emissions and global warming impact of three biomass cookstoves in Ghana. Energies 2017, 10, 1-14. [CrossRef]

26. Jetter, J.; Zhao, Y.; Smith, K.R.; Khan, B.; Yelverton, T.; Decarlo, P.; Hays, M.D. Pollutant emissions and energy efficiency under controlled conditions for household biomass cookstoves and implications for metrics useful in setting international test standards. Environ. Sci. Technol. 2012, 46, 10827-10834. [CrossRef] [PubMed]

27. Lombardi, F.; Riva, F.; Bonamini, G.; Barbieri, J.; Colombo, E. Laboratory protocols for testing of Improved Cooking Stoves (ICSs): A review of state-of-the-art and further developments. Biomass Bioenergy 2017, 98, 321-335. [CrossRef]

28. Global Alliance for Clean Cookstoves. Water Boiling Test, version 4.2.3; Global Alliance for Clean Cookstoves: Washington, DC, USA, 2014.

29. ISO. IWA 11:2012(en) Guidelines for Evaluating Cookstove Performance; ISO: Geneva, Switzerland, 2012.

30. Lombardi, F.; Riva, F.; Colombo, E. Guidelines for Reporting and Analysing Laboratory Test Results for Biomass Cooking Stoves; Politecnico di Milano: Milano, Italy, 2017.

31. Lombardi, F.; Riva, F.; Colombo, E. Dealing with small sets of laboratory test replicates for Improved Cooking Stoves (ICSs): Insights for a robust statistical analysis of results. Biomass Bioenergy 2018, 115, 27-34. [CrossRef]

32. Riva, F.; Lombardi, F.; Pavarini, C.; Colombo, E. Fuzzy interval propagation of uncertainties in experimental analysis for improved and traditional three-Stone fire cookstoves. Sustain. Energy Technol. Assess. 2016, 18, 59-68. [CrossRef]

33. Wang, Y.; Sohn, M.D.; Wang, Y.; Lask, K.M.; Kirchstetter, T.W.; Gadgil, A.J. How many replicate tests are needed to test cookstove performance and emissions?-Three is not always adequate. Energy Sustain. Dev. 2014, 20, 21-29. [CrossRef]

34. Vianello, M.; Okello, S. The Energy Situation in Goudoubo Refugee Camp, Burkina Faso; Practical Action: London, UK, 2016.

(C) 2018 by the authors. Licensee MDPI, Basel, Switzerland. This article is an open access article distributed under the terms and conditions of the Creative Commons Attribution (CC BY) license (http:/ / creativecommons.org/licenses/by/4.0/). 